\title{
NEHARI'S PROBLEM AND COMPETING SPECIES SYSTEMS 㐫
}

\section{UN PROBLÈME DE NEHARI ET LES ESPÈCES EN COMPETITION}

\author{
M. CONTI, S. TERRACINI, G. VERZINI \\ Dipartimento di Matematica, Politecnico di Milano, P.zza L. da Vinci, 32, 20133 Milano (I), Italy
}

Received 9 October 2000, revised 28 September 2001

ABSTRACT. - We consider an optimal partition problem in $\mathrm{N}$-dimensional domains related to a method introduced by Nehari [22]. We prove existence of the minimal partition and some extremality conditions. Moreover we show some connections between the variational problem, the behaviour of competing species systems with large interaction and changing sign solutions to elliptic superlinear equations.

(c) 2002 L'Association Publications de l'Institut Henri Poincaré. Published by Elsevier B.V. All rights reserved

RÉSUMÉ. - Nous étudions un problème de partition optimale dans $\mathbb{R}^{N}$ qui se relie à une métode introduite par Nehari [22]. Nous établissons l'existence de la partition optimale et quelques conditions d'extremalité. En plus nous démontrons un lien entre le problème variationel, le comportement asymptotique de certains systèmes quand l'interaction tends à l'infini et les solutions qui changent de signe pour les equations elliptiques surlinéaires.

(C) 2002 L'Association Publications de l'Institut Henri Poincaré. Published by Elsevier B.V. All rights reserved

AMS classification: $35 \mathrm{~J} 65 ; 58 \mathrm{E} 05$

\section{Introduction}

In a pioneering paper of the sixties Z. Nehari, [22], proposed a method for finding solutions with prescribed number of zeroes to ordinary differential boundary value problems. In spite of its simplicity, Nehari's method has revealed very useful in the search of changing sign solutions to superlinear second order ODEs and radially symmetric PDEs (see [3] and references therein).

In this paper we are concerned with the following related problem: let $\Omega \subset \mathbb{R}^{N}$ $(N \geqslant 1)$ be a smooth bounded domain and $f \in C([0, \infty))$ be superquadratic according

\footnotetext{
Work partially supported by MURST, Project "Metodi Variazionali ed Equazioni Differenziali Non Lineari".
} 
to [22] (see $\left(f_{2}\right)$ below). Set $F(s)=\int_{0}^{s} f(t) \mathrm{d} t$ and let us consider the following functional defined for all $u \in H_{0}^{1}(\Omega)$

$$
J^{*}(u):=\int_{\Omega}\left(\frac{1}{2}|\nabla u(x)|^{2}-F(u(x))\right) \mathrm{d} x ;
$$

let us introduce the set function

$$
\varphi(\omega):=\inf _{\substack{w \in H^{1}(\omega) \\ w>0}} \sup _{\lambda>0} J^{*}(\lambda w),
$$

where $\omega \subset \Omega$ is an open subset of $\Omega$. We are concerned with the problem of finding $a$ partition of $\Omega$ that achieves

$$
\inf \left\{\varphi\left(\omega_{1}\right)+\varphi\left(\omega_{2}\right): \overline{\omega_{1} \cup \omega_{2}}=\bar{\Omega}, \omega_{1} \cap \omega_{2}=\emptyset\right\} .
$$

When $N=1$ (or when dealing with the radially symmetric case) the existence of an optimal partition can be easily obtained. Moreover, as shown in [3] through the analysis of the extremality condition involved in (1), the minimal pair provides the supports of the positive and negative parts of a changing sign solution to the differential equation associated to $J^{*}$. This procedure is precisely what is nowadays called Nehari's method, and thus in the following we will refer to (1) as Nehari's problem in the $N$-dimensional case. In this paper we are interested in two main questions, namely the actual solvability of (1) when $N \geqslant 2$, and then the determination of the extremality conditions provided by the minimization procedure.

As a first step we will face the minimization in a relaxed sense, and we will establish in Theorem 2.2 that (1) is achieved by a pair $\left(\omega_{1}, \omega_{2}\right)$ where $\omega_{i}$ are the disjoint supports of nonnegative functions $u_{0}$ and $v_{0}$ belonging to $H_{0}^{1}(\Omega)$ - thus not necessarily open.

The proof we provide for Theorem 2.2 shows a somewhat surprising connection with the analysis of the segregation phenomena in population dynamics with diffusion and large interaction. This analysis has been carried out in the last two decades in many works, see e.g. $[6,19,20]$, and in particular by E.N. Dancer and others (see $[8-13,15-$ 18]). These authors mainly deal with Lotka-Volterra type systems, that is, a class of nonvariational superlinear elliptic systems. In the present paper we study a complementary class of systems, including

$$
\begin{cases}-\Delta u(x)=f(u(x))-\frac{1}{\varepsilon} \frac{\partial}{\partial u} G(u(x), v(x)) & \text { in } \Omega \\ -\Delta v(x)=f(v(x))-\frac{1}{\varepsilon} \frac{\partial}{\partial v} G(u(x), v(x)) & \text { in } \Omega \\ (u, v) \in H_{0}^{1}(\Omega) \times H_{0}^{1}(\Omega) & \text { in } \Omega, \\ u>0, \quad v>0 & \end{cases}
$$

where $\varepsilon>0$ and $G$ is positive and such that $G(s, t)=0$ iff $s \cdot t=0$. A system of this type can be seen as a variational version of a stationary case in a model of the 
interactive competition between two populations, and the problem of the existence of solutions for (2) is of independent interest. We will face the question for more general nonlinearities and we will provide the existence result Theorem 2.1 by developing a Nehari type procedure for elliptic systems; as an application it will follow that for every $\varepsilon>0$ (2) has a solution $\left(u_{\varepsilon}, v_{\varepsilon}\right)$. Next in Section 4 we study the asymptotic behavior of $u_{\varepsilon}$ and $v_{\varepsilon}$ as $\varepsilon \rightarrow 0$; roughly speaking, as the competing term becomes larger and larger, the two populations $u_{\varepsilon}, v_{\varepsilon}$ undergo a segregation, and this process leads to a final configuration $\left(u_{0}, v_{0}\right)$ where the populations are in fact separated. It is worth pointing out that this is one of the phenomena already observed in [17], although the internal dynamics of the systems therein are opposite to ours and the technical reasons for this behavior are completely different.

We proceed our analysis with the intent to prove that the partition $\left(\operatorname{supp}\left(u_{0}\right), \operatorname{supp}\left(v_{0}\right)\right)$ is in fact a solution of the original Nehari's problem (1). As a first result we obtain from the asymptotic analysis that $u_{0}$ and $v_{0}$ solve the differential equation

$$
-\Delta w(x)=f(w(x))
$$

each on its support. In fact, a much stronger result holds in the form of Theorem 2.3, where we show that the function $w_{0}:=u_{0}-v_{0}$ is a solution of (3) - when $f$ is extended as an odd function on $\mathbb{R}$. Now the regularity theory for elliptic PDEs implies that each solution to (3), and in particular $w_{0}$, must be smooth; as immediate consequence the supports of both $u_{0}$ and $v_{0}$ are open and we finally have an optimal partition to the Nehari's problem in the form $\left(\operatorname{supp}\left(u_{0}\right), \operatorname{supp}\left(v_{0}\right)\right)$. Moreover, the regularity of $w_{0}$ also proves the smoothness of the free boundary and proves that the extremality condition is

$$
\nabla u_{0}(x)=-\nabla v_{0}(x) \quad x \in \partial\left(\operatorname{supp}\left(u_{0}\right)\right) \cap \partial\left(\operatorname{supp}\left(v_{0}\right)\right),
$$

in analogy with the one-dimensional case. With these information the analysis of the $N$-dimensional Nehari's problem in the case of partitions of $\Omega$ into $k=2$ subsets is complete.

As a final remark let us mention that the natural extension of the Nehari's problem we are facing, consists in trying to partition $\Omega$ in $k \geqslant 3$ subsets: this seems to be a more delicate problem that will be the object of a forthcoming paper.

\section{Assumptions and main results}

In this paper we shall deal with nonlinearities $f: \mathbb{R} \rightarrow \mathbb{R}$ and $H: \mathbb{R}^{2} \rightarrow \mathbb{R}^{+}$satisfying the following set of assumptions:

$\left(f_{1}\right) f$ is $C^{1}$ and there exist positive constants $C, p$ such that for all $s \in \mathbb{R}$ and a.e. $x \in \Omega$

$$
|f(x, s)| \leqslant C\left(1+|s|^{p-1}\right) \quad 2<p<2^{*},
$$

where $2^{*}=+\infty$ when $N=2$ and $2^{*}=2 N /(N-2)$ when $N \geqslant 3$;

$\left(f_{2}\right)$ there exists $\gamma>0(2+\gamma \leqslant p)$ such that, for all $s \neq 0$ and a.e $x \in \Omega$

$$
f_{s}(x, s) s^{2}-(1+\gamma) f(x, s) s>0 ;
$$


$\left(h_{1}\right) H$ is $C^{1}\left(\mathbb{R}^{2}\right)$ and $C^{2}$ on $\mathbb{R}^{2} \backslash\{(s, 0),(0, t): s, t \in \mathbb{R}\}$; there exists a positive constant $0<\beta<\gamma$ such that

$$
|H(x, s, t)| \leqslant C(1+|s|+|t|)^{2+\beta} ;
$$

$\left(h_{2}\right)$ there exists $0<\alpha \leqslant \beta$ such that the Hessian matrix

$$
\left(\begin{array}{cc}
H_{s s}(s, t) s^{2}-(1+\alpha) H_{s}(s, t) s & H_{s t}(s, t) s t \\
H_{s t}(s, t) s t & H_{t t}(s, t) t^{2}-(1+\alpha) H_{t}(s, t) t
\end{array}\right)
$$

is non-positive definite for all $(s, t)$ such that $s \neq 0, t \neq 0$ (i.e. positive (or zero) $\left(h_{3}\right)$ determinant and negative diagonal terms);

$$
\begin{gathered}
H(s, t) \geqslant 0, H_{s}(s, t) s \geqslant 0, H_{t}(s, t) t \geqslant 0 \quad \text { if } s t \neq 0, \\
H(s, t)=H_{s}(s, t)=H_{t}(s, t)=0 \quad \text { if } s t=0 .
\end{gathered}
$$

Our first result, discussed in Section 3, is concerned with systems having a competitive interaction:

THEOREM 2.1. - Let $f$ satisfy $\left(f_{1}\right),\left(f_{2}\right)$ and $H$ satisfy $\left(h_{1}\right)-\left(h_{3}\right)$. Then there exists a solution $(u, v) \in H_{0}^{1}(\Omega) \times H_{0}^{1}(\Omega)$ to the problem

$$
\begin{cases}-\Delta u(x)=f(u(x))-\frac{\partial}{\partial u} H(u(x), v(x)) & \text { in } \Omega \\ -\Delta v(x)=f(v(x))-\frac{\partial}{\partial v} H(u(x), v(x)) & \text { in } \Omega \\ u>0, \quad v>0 & \text { in } \Omega .\end{cases}
$$

Next in Section 4 we turn to the Nehari's problem. More precisely we deal with the following relaxed formulation of (1): let us define the functional

$$
J_{0}(u, v):=\int_{\Omega}\left(\frac{1}{2}|\nabla u(x)|^{2}+\frac{1}{2}|\nabla v(x)|^{2}-F(u(x))-F(v(x))\right) \mathrm{d} x
$$

and let us consider the question of finding a pair of positive functions, $u_{0}$ and $v_{0}$, having disjoint support and realizing $J\left(u_{0}, v_{0}\right)=d_{0}$ :

$$
d_{0}:=\inf _{\substack{u, v \in H_{0}^{1}(\Omega) \\ u \neq 0,0 \neq 0\\}} \sup _{\substack{\lambda>0 \\ \mu>0}} J_{0}(\lambda u, \mu v)
$$

We shall prove

THEOREM 2.2. - Under assumptions $\left(f_{1}\right),\left(f_{2}\right)$ problem $\left(P_{0}\right)$ has a solution $\left(u_{0}, v_{0}\right) \in$ $H_{0}^{1}(\Omega) \times H_{0}^{1}(\Omega)$ such that $u_{0}>0, v_{0}>0 ;$ moreover $\operatorname{supp}\left(u_{0}\right) \cup \operatorname{supp}\left(v_{0}\right)$ is a relaxed solution of (1). 
Section 4 will be devoted to prove this result, with arguments crucially based on the validity of Theorem 2.1 .

In the last Section 5 we study the existence of changing sign solutions to the elliptic equation (3); the main result we obtain is

THEOREM 2.3. - Under assumptions $\left(f_{1}\right),\left(f_{2}\right)$ the function $w_{0}:=u_{0}-v_{0}$ is solution to the problem

$$
\left\{\begin{array}{l}
-\Delta w(x)=f(w(x)) \quad \text { in } \Omega \\
w \in H_{0}^{1}(\Omega) \\
w \text { changes sign exactly one time in } \Omega .
\end{array}\right.
$$

Moreover the pair $\operatorname{supp}\left(u_{0}\right) \cup \operatorname{supp}\left(v_{0}\right)$ is a solution of (1).

The existence of a changing sign solution is a well known fact, also for more general kinds of elliptic equations (see $[23,1,2,5,7,8,14]$ ). The remarkable fact in our context is the particular form of the solution we find, since, as we have already remarked in the introduction, it provides both the solvability of the original Nehari's problem (1) and the extremality condition (4), besides the regularity of the free boundary.

\section{A class of elliptic system}

Aim of this section is to prove Theorem 2.1; to this end we assume that $\left(f_{1}\right),\left(f_{2}\right)$, and $\left(h_{1}\right)-\left(h_{3}\right)$ hold and we wish to provide the existence of a pair $(u, v) \in H_{0}^{1}(\Omega) \times H_{0}^{1}(\Omega)$ where both $u$ and $v$ are positive and solve (5). We seek solution of (5) as critical points of the following functional

$$
\begin{aligned}
J(u, v):= & \int_{\Omega}\left(\frac{1}{2}|\nabla u(x)|^{2}+\frac{1}{2}|\nabla v(x)|^{2}-F(u(x))\right. \\
& -F(v(x))+H(u(x), v(x))) \mathrm{d} x
\end{aligned}
$$

(here, with some abuse of notation, we extend $F$ and $H$ by symmetry: $F(s):=F(|s|)$, $H(s, t):=H(|s|,|t|))$; note that the extended functions satisfy the same assumptions than the original ones, thus $J \in C^{1}\left(H_{0}^{1}(\Omega) \times H_{0}^{1}(\Omega), \mathbb{R}\right)$ and its critical points in $H_{0}^{1}(\Omega) \times H_{0}^{1}(\Omega)$ are exactly the classical solutions to (5). Furthermore, $F$ and $H$ are even with respect to each of their variables and this further property will be used to obtain solutions to (5) which are indeed positive.

In particular we are interested in the search of minimal solutions of (5), that is, critical points of $J$ achieving the lower value of $J$ when restricted to the critical set $\mathcal{N}$ :

$$
\mathcal{N}:=\left\{(u, v) \in \mathcal{T}: \frac{\partial J(u, v)}{\partial u} \cdot u=\frac{\partial J(u, v)}{\partial v} \cdot v=0\right\},
$$

where

$$
\mathcal{T}:=\left\{(u, v) \in H_{0}^{1}(\Omega) \times H_{0}^{1}(\Omega): u \not \equiv 0, v \not \equiv 0\right\} .
$$


Setting

$$
c:=\inf _{(u, v) \in \mathcal{N}} J(u, v)
$$

we are going to prove

PROPOSITION 3.1. - Under assumptions $\left(f_{1}\right)-\left(h_{3}\right)$ there exists a critical point for $J$, say $\left(u_{c}, v_{c}\right) \in \mathcal{N}$, such that $J\left(u_{c}, v_{c}\right)=c$.

The proof of the proposition will be divided into three steps; the first consists in studying the behaviour of $J$ when restricted to the plane generated by a fixed pair $(u, v)$ with nontrivial components:

LEMMA 3.1. - Let $(u, v) \in \mathcal{T}$ and consider

$$
\Phi(u, v):=\sup _{\substack{\lambda>0 \\ \mu>0}} J(\lambda u, \mu v) .
$$

Then there exists a unique pair of positive numbers $\lambda(u, v)$ and $\mu(u, v)$ such that

(i) $\Phi(u, v)=J(\lambda(u, v) u, \mu(u, v) v)$;

(ii) $(\lambda(u, v) u, \mu(u, v) v) \in \mathcal{N}$;

(iii) there exists $\gamma, \gamma_{1}>0$ such that if $(u, v) \in \mathcal{N}$ then $\|u\|_{p} \geqslant \gamma,\|v\|_{p} \geqslant \gamma$ and $\|u\| \geqslant \gamma_{1},\|v\| \geqslant \gamma_{1}$

(iv) $\Phi(\cdot)$ is l.s.c. with respect to the weak convergence in $\mathcal{T}$;

(v) the map $(u, v) \mapsto(\lambda(u, v), \mu(u, v))$ is continuous from $\mathcal{T}$ to $\mathbb{R}^{+} \times \mathbb{R}^{+}$.

Proof. - Fix $(u, v) \in \mathcal{T}$ and define $\Psi(\lambda, \mu):=J(\lambda u, \mu v)$. As a function of two real variables, $\Psi$ is of class $C^{2}$ on $\mathbb{R}^{2}$ but the axes. We are interested in studying the set of critical points of $\Psi$; by a direct analysis, exploiting the superlinear behaviour of $f$ as in $\left(f_{2}\right)$ and the positivity of $H$ as in $\left(h_{3}\right)$, it is easy to show that $\Psi$ has a local minimum at the origin and one mountain pass on each of the semi-axes. On the other hand, by means of topological degree computations, we will show that there are exactly four local maxima, one in each quadrant, and this will finally prove assertion (i).

Let $(\bar{\lambda}, \bar{\mu})$ be a critical point of $\Psi$; then

$$
\begin{aligned}
& \bar{\lambda} \int_{\Omega}\left(|\nabla u|^{2}-\frac{1}{\bar{\lambda}^{2}} f(\bar{\lambda} u) \bar{\lambda} u+\frac{1}{\bar{\lambda}^{2}} H_{u}(\bar{\lambda} u, \bar{\mu} v) \bar{\lambda} u\right)=0, \\
& \bar{\mu} \int_{\Omega}\left(|\nabla v|^{2}-\frac{1}{\bar{\mu}^{2}} f(\bar{\mu} v) \bar{\lambda} v+\frac{1}{\bar{\mu}^{2}} H_{v}(\bar{\lambda} u, \bar{\mu} v) \bar{\mu} v\right)=0 .
\end{aligned}
$$

Claim 1. - If $\bar{\lambda} \neq 0$ and $\bar{\mu} \neq 0$, then $(\bar{\lambda}, \bar{\mu})$ is a local maximum for $\Psi$.

Let $\bar{\lambda} \neq 0$ and $\bar{\mu} \neq 0$. In order to investigate the nature of such a critical point of $\Psi$, we are going to study the quadratic form associated to the pair $(\bar{\lambda}, \bar{\mu})$, defined as

$$
Q(a, b)=\Psi_{\lambda \lambda}(\bar{\lambda}, \bar{\mu}) a^{2}+2 \Psi_{\lambda \mu}(\bar{\lambda}, \bar{\mu}) a b+\Psi_{\mu \mu}(\bar{\lambda}, \bar{\mu}) b^{2}, \quad(a, b) \in \mathbb{R}^{2} ;
$$

we wish to show that $Q(a, b)<0$ for all $(a, b) \in \mathbb{R}^{2}$. We estimate the second derivative $\Psi_{\lambda \lambda}$ by exploiting first $\left(f_{2}\right)$ and then the identities (8) 


$$
\begin{aligned}
\Psi_{\lambda \lambda}(\bar{\lambda}, \bar{\mu}) & =\int_{\Omega}\left(|\nabla u|^{2}-\frac{1}{\bar{\lambda}^{2}} f^{\prime}(\bar{\lambda} u) \bar{\lambda}^{2} u^{2}+\frac{1}{\bar{\lambda}^{2}} H_{u u}(\bar{\lambda} u, \bar{\mu} v) \bar{\lambda}^{2} u^{2}\right) \\
& \leqslant \int_{\Omega}\left(|\nabla u|^{2}-\frac{1+\alpha}{\bar{\lambda}^{2}} f(\bar{\lambda} u) \bar{\lambda} u+\frac{1}{\bar{\lambda}^{2}} H_{u u}(\bar{\lambda} u, \bar{\mu} v) \bar{\lambda}^{2} u^{2}\right) \\
& =-\alpha \int_{\Omega}|\nabla u|^{2}+\frac{1}{\bar{\lambda}^{2}} \int_{\Omega}\left(H_{u u}(\bar{\lambda} u, \bar{\mu} v) \bar{\lambda}^{2} u^{2}-(1+\alpha) H_{u}(\bar{\lambda} u, \bar{\mu} v) \bar{\lambda} u\right) .
\end{aligned}
$$

Analogous calculations lead to:

$$
\Psi_{\mu \mu}(\bar{\lambda}, \bar{\mu}) \leqslant-\alpha \int_{\Omega}|\nabla v|^{2}+\frac{1}{\bar{\mu}^{2}} \int_{\Omega}\left(H_{v v}(\bar{\lambda} u, \bar{\mu} v) \bar{\mu}^{2} v^{2}-(1+\alpha) H_{v}(\bar{\lambda} u, \bar{\mu} v) \bar{\mu} v\right),
$$

while

$$
\Psi_{\mu \lambda}(\bar{\lambda}, \bar{\mu})=\frac{1}{\bar{\mu} \bar{\lambda}} H_{u v}(\bar{\lambda} u, \bar{\mu} v) \bar{\lambda} u \bar{\mu} v .
$$

Thus we can bound the form $Q$ from above with

$$
Q(a, b) \leqslant-\alpha a^{2} \int_{\Omega}|\nabla u|^{2}-\alpha b^{2} \int_{\Omega}|\nabla v|^{2}+Q_{H}(\bar{\lambda} u, \bar{\mu} v)(a, b),
$$

where the quadratic form $Q_{H}$ is negatively definite by assumption $\left(h_{2}\right)$. Thus the claim follows.

CLAIM 2. - There are exactly four critical points with nonzero components (and thus maxima), one in each of the quadrants.

By the growth assumptions on $F$ and $H, \Psi(\lambda, \mu) \rightarrow-\infty$ as $|\lambda|+|\mu| \rightarrow \infty$, implying that $\Psi$ must have at least a local maximum in each of the quadrants. Moreover, since $\Psi$ turns out to be concave outside a suitably large ball $B_{R}$, we have

$$
\operatorname{deg}\left(\nabla \Psi, 0, B_{R}\right)=+1
$$

On the other hand, let us count all the possible critical points of $\Psi$ : by the superquadraticity property of $F$ we know that $\Psi$ has one local minimum at the origin, providing a local degree +1 ; moreover, by arguments similar to the ones in the previous Claim 1, it is easy to prove that $\Psi$ has exactly four critical points with one null component (one on each semiaxis) having index -1 . A word of caution must be entered at this point: by the assumptions on $H, J$ needs not to be $C^{2}$ on the axis; this problem can be avoided by an homotopy argument. Finally the other possible critical points are local maxima by Claim 1, and thus the local degree at each of them is +1 . Let $n$ be the number of such maxima: by the excision property of the degree it must hold:

$$
+1=\operatorname{deg}\left(\nabla \Psi, 0, B_{R}\right)=(+1) \cdot 1+(-1) \cdot 4+(+1) \cdot n
$$

that gives $n=4$, proving the claim. 
Now we define $(\lambda(u, v), \mu(u, v))$ as the unique local maximum of $\Psi$ in the first quadrant, and we will prove that it satisfies (i)-(v).

Assertion (i) follows by construction; (ii) follows by noting that $(\lambda(u, v), \mu(u, v))$ is (the unique) positive solution of (8). Now take $(u, v) \in \mathcal{N}$, so that $\lambda(u, v)=\mu(u, v)=1$ and by (8) it holds, for instance

$$
0=\int_{\Omega}\left(|\nabla u|^{2}-f(u) u+H_{u}(u, v) u\right) \geqslant \int_{\Omega}\left(|\nabla u|^{2}-f(u) u\right)
$$

by the positivity of $H$. Now (iii) follows by using the subcritical assumption $\left(f_{1}\right)$ and the Sobolev inequality.

In order to prove the weak 1.s.c. property of $\Phi$, let $\left(u_{n}, v_{n}\right)$ weakly converging to $\left(u_{0}, v_{0}\right) \in \mathcal{T}$. Then $\left\|u_{0}\right\|+\left\|v_{0}\right\| \leqslant \liminf \left\|u_{n}\right\|+\left\|v_{n}\right\|$; on the other side, due to the subcritical growth of $F$ (assumption $\left(f_{1}\right)$ ) and thanks to the compact embedding $H_{0}^{1}(\Omega) \subset L^{q}$ for all $2<q<2^{*}$, it holds

$$
\begin{aligned}
\Phi\left(u_{0}, v_{0}\right) & =J\left(\lambda\left(u_{0}, v_{0}\right) u_{0}, \mu\left(u_{0}, v_{0}\right) v_{0}\right) \\
& \leqslant J\left(\lambda\left(u_{0}, v_{0}\right) u_{n}, \mu\left(u_{0}, v_{0}\right) v_{n}\right)+\mathrm{o}(1) \leqslant \Phi\left(u_{n}, v_{n}\right)+\mathrm{o}(1),
\end{aligned}
$$

and passing to the limit

$$
\Phi\left(u_{0}, v_{0}\right) \leqslant \liminf _{n \rightarrow \infty} \Phi\left(u_{n}, v_{n}\right)
$$

as required. Finally (v) holds as a consequence of the Implicit Function Theorem applied to $(8)$.

Note that, if we define the following value

$$
d:=\inf _{(u, v) \in \mathcal{T}} \Phi(u, v)
$$

it turns out by the lemma that $d=c$. This remark will be useful in proving that the problem of minimizing $J$ on the Nehari manifold $\mathcal{N}$ has a solution:

LEMMA 3.2. - There exists $\left(u_{c}, v_{c}\right) \in \mathcal{N}$ such that $J\left(u_{c}, v_{c}\right)=c$.

Proof. - Let $\left(u_{n}, v_{n}\right) \subset \mathcal{N}$ be a minimizing sequence and assume $J\left(u_{n}, v_{n}\right) \leqslant c+1$. Now, computing: $(\alpha+2) J\left(u_{n}, v_{n}\right)-\nabla J\left(u_{n}, v_{n}\right) \cdot u_{n}$ with $\alpha>0$ as in assumption $\left(h_{2}\right)$, we have

$$
\begin{aligned}
(\alpha+2) c \geqslant & \frac{\alpha}{2} \int_{\Omega}\left[\left|\nabla u_{n}\right|^{2}+\left|\nabla v_{n}\right|^{2}\right]+\int_{\Omega}\left[f\left(u_{n}\right) u_{n}-(2+\alpha) F\left(u_{n}\right)\right] \\
& +\int_{\Omega}\left[f\left(v_{n}\right) v_{n}-(2+\alpha) F\left(v_{n}\right)\right] \\
& +\int_{\Omega}\left[(2+\alpha) H\left(u_{n}, v_{n}\right)-H_{u}\left(u_{n}, v_{n}\right) u_{n}-H_{v}\left(u_{n}, v_{n}\right) v_{n}\right],
\end{aligned}
$$


where by assumptions $\left(f_{2}\right)$ and $\left(h_{2}\right)$ the three terms in square brackets are positive. We have

$$
\left\|u_{n}\right\|^{2}+\left\|v_{n}\right\|^{2} \leqslant \frac{2(2+\alpha)}{\alpha}(c+1)
$$

and thus the sequence $\left(u_{n}, v_{n}\right)$ is bounded. Hence there exists $(\bar{u}, \bar{v}) \in H_{0}^{1}(\Omega) \times H_{0}^{1}(\Omega)$ weak limit of $\left(u_{n}, v_{n}\right)$. It is easy to verify that $(\bar{u}, \bar{v}) \in \mathcal{T}$; indeed by Lemma 3.1(iii), it holds $\left\|u_{n}\right\|_{p} \geqslant \gamma,\left\|v_{n}\right\|_{p} \geqslant \gamma$ for all $n \in \mathbb{N}$, and, by the strong convergence in $L^{p}$ of $\left(u_{n}, v_{n}\right)$ to $(\bar{u}, \bar{v})$, we also obtain $\|\bar{u}\|_{p} \geqslant \gamma,\|\bar{v}\|_{p} \geqslant \gamma$.

Now we can apply Lemma 3.1 to $(\bar{u}, \bar{v})$ : by the weak 1.s.c. property of $\Phi$ as in (iv), we have $\Phi(\bar{u}, \bar{v}) \leqslant c$; furthermore, by definition of $d$ as in (10) it holds $\Phi(\bar{u}, \bar{v}) \geqslant d \equiv c$. Thus

$$
c \equiv \sup _{\substack{\lambda>0 \\ \mu>0}} J(\lambda \bar{u}, \mu \bar{v})
$$

and by applying Lemma 3.1 again, we obtain the existence of a pair $(\bar{\lambda}, \bar{\mu})$ such that (due to (i)) $c \equiv J(\bar{\lambda} \bar{u}, \bar{\mu} \bar{v})$ and (due to (ii)) $(\bar{\lambda} \bar{u}, \bar{\mu} \bar{v}) \in \mathcal{N}$. Setting $\left(u_{c}, v_{c}\right):=(\bar{\lambda} \bar{u}, \bar{\mu} \bar{v})$ we finally conclude the proof.

LEMMA 3.3. - If $\left(u_{c}, v_{c}\right) \in \mathcal{N}$ and $J\left(u_{c}, v_{c}\right)=c$, then $\left(u_{c}, v_{c}\right)$ is a critical point of $J$.

Proof. - Assume by contradiction that $\left(u_{c}, v_{c}\right) \in \mathcal{N}, J\left(u_{c}, v_{c}\right)=c$, but $\nabla J\left(u_{c}, v_{c}\right) \neq$ 0 . Then there exists $\rho>0$ and $\delta>0$ (we may assume $4 \delta<\gamma_{1}$ as in Lemma 3.1(iii)) such that

$$
(u, v) \in B_{\delta}\left(\left(u_{c}, v_{c}\right)\right) \Rightarrow\|\nabla J(u, v)\| \geqslant \rho .
$$

By the quantitative deformation lemma (see, for instance, [24]) we derive the existence of a continuous map $\eta: H_{0}^{1}(\Omega) \times H_{0}^{1}(\Omega) \rightarrow H_{0}^{1}(\Omega) \times H_{0}^{1}(\Omega)$ and a constant $v>0$ such that:

(i) $\eta(u, v)=(u, v)$ for all $(u, v)$ such that $\left\|\left(u-u_{c}, v-v_{c}\right)\right\|>4 \delta$ or $|J(u, v)-c|>$ $2 v$

(ii) $J(\eta(u, v)) \leqslant J(u, v)$ for all $(u, v) \in H_{0}^{1}(\Omega) \times H_{0}^{1}(\Omega)$;

(iii) $(u, v) \in B_{2 \delta}\left(\left(u_{c}, v_{c}\right)\right) \wedge J(u, v) \leqslant c+v \Rightarrow J(\eta(u, v)) \leqslant c-v$.

Let us consider the deformation under this map of the surface $\Gamma(s, t):=\left(s R u_{c}, t R v_{c}\right)$, where $R$ is fixed in such a way that $(R-1) \gamma_{1}>4 \delta$. By the monotonicity properties of $\eta$ and Lemma 3.1, we have that $\sup _{s>0, t>0} \eta(\Gamma(s, t))<c$. Now we claim that $\eta \circ \Gamma$ intersects the manifold $\mathcal{N}$ and thus, by definition of $c$ it holds $\sup \eta(\Gamma(s, t)) \geqslant c$, a contradiction. To prove the claim, let us consider the map:

$$
\begin{aligned}
\mathcal{H}:[0,1]^{2} & \rightarrow \mathbb{R}^{+} \times \mathbb{R}^{+} \\
(s, t) & \mapsto(\lambda(\eta(\Gamma(s, t)))-1, \mu(\eta(\Gamma(s, t)))-1)
\end{aligned}
$$

which is continuous by Lemma 3.1(v). By construction it holds:

- if $s=0$, then $\mathcal{H}_{1}(0, \cdot)=+\infty$; indeed, since $\Gamma(0, t)=\left(0, t R v_{c}\right)$, it holds $\| \Gamma_{1}(0, t)-$ $u_{c}\|=\| u_{c} \|>\gamma_{1}>4 \delta$ by the choice of $\delta$. Thus by property (i) of the map $\eta$, we know that $\eta(\Gamma(0, t))=\Gamma(0, t)=$ and thus $\mathcal{H}_{1}(0, t)=\lambda\left(0, t R v_{c}\right)=+\infty$;

- if $s=1$, then $\mathcal{H}_{1}(0, \cdot)<0$; indeed since $\Gamma(1, t)=\left(R u_{c}, t R v_{c}\right)$, it holds $\| \Gamma_{1}(1, t)-$ $u_{c}\|=(R-1)\| u_{c} \|>4 \delta$ by the choice of $R$. Thus $\eta(\Gamma(1, t))=\Gamma(1, t)$ and 
$\mathcal{H}_{1}(1, t)=\lambda\left(R u_{c}, t R v_{c}\right)<1$. This last fact is due to the choice of $R$ large: observe that, due to the superquadraticity of $F, J$ is negative far from the origin; on the other hand, the functions $\lambda(\cdot, \cdot), \mu(\cdot, \cdot)$ provide the scaling that the arguments needs to achieve the maximum of $J$, that is positive and hence achieved near the origin. Analogous considerations allow to prove that the second component of $\mathcal{H}$ is positive when $t=0$ and negative if $t=1$. Thus we are in condition to apply Miranda Theorem [21], and we find $(\bar{s}, \bar{t})$ such that $\mathcal{H}(\bar{s}, \bar{t})=(0,0)$; this implies $\eta(\Gamma(\bar{s}, \bar{t})) \in \mathcal{N}$ and close the proof.

Now the proof of Proposition 3.1 follows by simply combining Lemma 3.2 and Lemma 3.3, and thus we have the existence of a pair $\left(u_{c}, v_{c}\right)$ of critical points of $J$ such that $J\left(u_{c}, v_{c}\right)=c$. What is left to show, in order to obtain by this result the full of Theorem 2.1, is that both $u_{c}$ and $v_{c}$ are strictly positive. To this aim we note that $J\left(u_{c}, v_{c}\right)=c=J\left(\left|u_{c}\right|,\left|v_{c}\right|\right)$ by definition of $\bar{F}$ and $\bar{H}$. Then by Lemma 3.3 we know that $\left(\left|u_{c}\right|,\left|v_{c}\right|\right)$ is a critical point of $J$ and so its components must be smooth by the standard regularity theory for elliptic PDEs. In this way we obtain the equalities $u_{c}=\left|u_{c}\right| \geqslant 0, v_{c}=\left|v_{c}\right| \geqslant 0$; now the strict inequalities comes by the strong maximum principle. With this final remark the proof of our main Theorem 2.1 is complete.

\section{The relaxed Nehari's problem}

We are going to deal with the relaxed formulation of Nehari's problem presented in Section 2 in the form $\left(P_{0}\right)$. Let us recall that it consists in facing the minimax problem

$$
d_{0}:=\inf _{\substack{u, v \in H_{0}^{1}(\Omega) \\ u \neq 0, v \neq 0}} \sup _{\substack{\lambda>0 \\ \mu>0}} J_{0}(\lambda u, \mu v),
$$

where

$$
J_{0}(u, v):=\int_{\Omega}\left(\frac{1}{2}|\nabla u(x)|^{2}+\frac{1}{2}|\nabla v(x)|^{2}-F(u(x))-F(v(x))\right) \mathrm{d} x .
$$

Note that $d_{0}$ coincides with the minimal value of $J_{0}$ restricted to the critical set $\mathcal{N}_{0}$

$$
\begin{aligned}
& c_{0}:=\inf \left\{J_{0}(u, v):(u, v) \in \mathcal{N}_{0}, \int u^{2} v^{2}=0\right\}, \\
& \mathcal{N}_{0}:=\left\{(u, v) \in \mathcal{T}: \frac{\partial J_{0}(u, v)}{\partial u} \cdot u=\frac{\partial J_{0}(u, v)}{\partial v} \cdot v=0\right\} .
\end{aligned}
$$

Indeed, as consequence of Lemma 3.1 (note that $H \equiv 0$ satisfies assumptions $\left(h_{1}\right)-\left(h_{3}\right)$ ) it holds $d_{0}=c_{0}$.

Our main result in this setting is Theorem 2.2, that is, the existence of positive functions $u_{0}, v_{0}$, having disjoint supports and realizing $J_{0}\left(u_{0}, v_{0}\right)=d_{0}$. We present a proof of these facts that passes through the solution of the approximating problems $\left(P_{\varepsilon}\right)$ described below. 


\subsection{Systems with large interaction}

Let us fix a function $G: \mathbb{R}^{2} \rightarrow \mathbb{R}$ satisfying $\left(h_{1}\right)-\left(h_{3}\right)$. For all fixed $\varepsilon>0$, let us define the energy functional associated to system (2) for such a choice of $G$ and $\varepsilon$ :

$$
\begin{aligned}
J_{\varepsilon}(u, v):= & \int_{\Omega}\left(\frac{1}{2}|\nabla u(x)|^{2}+\frac{1}{2}|\nabla v(x)|^{2}\right. \\
& \left.-F(u(x))-F(v(x))+\frac{1}{\varepsilon} G(u(x), v(x))\right) \mathrm{d} x
\end{aligned}
$$

and consider the following problem: finding a pair of positive functions, $u_{\varepsilon}$ and $v_{\varepsilon}$, which realize

$$
d_{\varepsilon}:=\inf _{\substack{u, v \in H_{0}^{1}(\Omega) \\ u \neq 0, v \neq 0}} \sup _{\substack{\lambda>0 \\ \mu>0}} J_{\varepsilon}(\lambda u, \mu v)
$$

Note that

$$
d_{\varepsilon} \leqslant d_{0}
$$

indeed $J_{\varepsilon}$ coincides with $J_{0}$ on pairs of functions having disjoint support.

We are going to prove

PROPOSITION 4.1. - Assume that $f$ satisfies $\left(f_{1}\right),\left(f_{2}\right)$. Then

1) for every $\varepsilon>0$ problem $\left(P_{\varepsilon}\right)$ has a solution $\left(u_{\varepsilon}, v_{\varepsilon}\right) \in H_{0}^{1}(\Omega) \times H_{0}^{1}(\Omega)$;

2) there exists $\left(u_{0}, v_{0}\right) \in H_{0}^{1}(\Omega) \times H_{0}^{1}(\Omega)$ such that $\left(u_{\varepsilon}, v_{\varepsilon}\right) \rightarrow\left(u_{0}, v_{0}\right)$ in $H_{0}^{1}(\Omega) \times$ $H_{0}^{1}(\Omega)$ and $\left(u_{0}, v_{0}\right)$ solves $\left(P_{0}\right)$.

Proof of 1). - Let $\varepsilon>0$ be fixed. By applying Theorem 2.1 with the choice $H(s, t):=$ $\frac{1}{\varepsilon} G(s, t)$, we immediately obtain the existence of a solution $\left(u_{\varepsilon}, v_{\varepsilon}\right)$ to $\left(P_{\varepsilon}\right)$. It is easy to prove that there exists $\gamma_{2}>0$, independent of $\varepsilon$, such that $\left\|u_{\varepsilon}\right\|+\left\|v_{\varepsilon}\right\|<\gamma_{2}$. To this end it suffices to recall inequality (11) in the proof of Lemma 3.2 that provides, for every $\varepsilon>0$,

$$
\left\|u_{\varepsilon}\right\|^{2}+\left\|v_{\varepsilon}\right\|^{2} \leqslant \frac{2(2+\alpha)}{\alpha}\left(c_{\varepsilon}+1\right) .
$$

Since $c_{\varepsilon}=d_{\varepsilon} \leqslant d_{0}$ the required estimate follows by setting

$$
\gamma_{2}:=\frac{2(2+\alpha)}{\alpha}\left(d_{0}+1\right)
$$

Henceforth there exists a weak limit $\left(u_{0}, v_{0}\right) \in H_{0}^{1}(\Omega) \times H_{0}^{1}(\Omega)$ :

$$
\left(u_{\varepsilon}, v_{\varepsilon}\right) \rightarrow\left(u_{0}, v_{0}\right) \quad \varepsilon \rightarrow 0 .
$$

Moreover it holds $u_{0} \not \equiv 0$ and $v_{0} \not \equiv 0$; indeed, as consequence of inequality (9) in the proof of Lemma 3.1(iii), we know that $\left\|u_{\varepsilon}\right\|_{p} \geqslant \gamma,\left\|v_{\varepsilon}\right\|_{p} \geqslant \gamma$ for some $\gamma>0$ independent of $\varepsilon$. By the compact embedding $H_{0}^{1}(\Omega) \hookrightarrow L^{p}$ we get $\left\|u_{0}\right\|_{p} \geqslant \gamma$ and $\left\|v_{0}\right\|_{p} \geqslant \gamma$, giving the result. 
The second part of Proposition 4.1 will be essentially provided in the following crucial lemma:

LEMmA 4.1. - Let $\left(u_{0}, v_{0}\right)$ be the weak limit of $\left(u_{\varepsilon}, v_{\varepsilon}\right)$ when $\varepsilon \rightarrow 0$ as in (15). Then it holds:

(i) $\left(u_{0}, v_{0}\right) \in \mathcal{N}_{0}$;

(ii) $\left\|u_{\varepsilon}-u_{0}\right\| \rightarrow 0,\left\|v_{\varepsilon}-v_{0}\right\| \rightarrow 0$;

(iii) $\frac{1}{\varepsilon} \int_{\Omega} G\left(u_{\varepsilon}, v_{\varepsilon}\right) \rightarrow 0$;

(iv) $c_{\varepsilon} \rightarrow c_{0}$.

Proof. - Let us first note that, since $\left(u_{0}, v_{0}\right) \in \mathcal{T}$, then we find $\lambda_{0}>0, \mu_{0}>0$ such that $\left(\lambda_{0} u_{0}, \mu_{0} v_{0}\right) \in \mathcal{N}_{0}$. By summing up the following equalities

$$
\begin{gathered}
\frac{\partial}{\partial u} J_{\varepsilon}\left(u_{\varepsilon}, v_{\varepsilon}\right) \cdot u_{\varepsilon}=0, \\
\frac{\partial}{\partial u} J_{0}\left(\lambda_{0} u_{0}, \mu_{0} v_{0}\right) \cdot \lambda_{0} u_{0}=0
\end{gathered}
$$

and then passing to the limit, we obtain

$$
\int_{\Omega}\left(\frac{f\left(\lambda_{0} u_{0}\right)}{\lambda_{0} u_{0}}-\frac{f\left(u_{0}\right)}{u_{0}}\right) u_{0}^{2}+\lim _{\varepsilon \rightarrow 0} \frac{1}{\varepsilon} \int_{\Omega} G_{u}\left(u_{\varepsilon}, v_{\varepsilon}\right) u_{\varepsilon} \leqslant 0 .
$$

Since $f(t) /|t|$ is increasing by $\left(f_{2}\right)$ and the limit is non-negative, we obtain that $\lambda_{0} \leqslant 1$. Analogous estimates for the component involving $v_{\varepsilon}$ and $v_{0}$ imply that $\mu_{0} \leqslant 1$ too.

Let us now make explicit the fact that $\left(\lambda_{0} u_{0}, \mu_{0} v_{0}\right) \in \mathcal{N}_{0}$ :

$$
\begin{aligned}
& \lambda_{0}^{2} \int_{\Omega}\left|\nabla u_{0}\right|^{2}-\int_{\Omega} f\left(\lambda_{0} u_{0}\right) \lambda_{0} u_{0}=0, \\
& \mu_{0}^{2} \int_{\Omega}\left|\nabla v_{0}\right|^{2}-\int_{\Omega} f\left(\mu_{0} v_{0}\right) \mu_{0} v_{0}=0, \\
& \frac{\lambda_{0}^{2}}{2} \int_{\Omega}\left|\nabla u_{0}\right|^{2}-\int_{\Omega} F\left(\lambda_{0} u_{0}\right)+\frac{\mu_{0}^{2}}{2} \int_{\Omega}\left|\nabla v_{0}\right|^{2}-\int_{\Omega} F\left(\mu_{0} v_{0}\right) \geqslant c_{0} .
\end{aligned}
$$

By multiplying the third inequality with $(2+\alpha), \alpha>0$ chosen as in assumption $\left(h_{2}\right)$, and then summing up with the two equalities, we obtain

$$
\begin{aligned}
(2+\alpha) c_{0} \leqslant & \frac{\lambda_{0}^{2} \alpha}{2} \int_{\Omega}\left|\nabla u_{0}\right|^{2}+\int_{\Omega}\left[f\left(\lambda_{0} u_{0}\right) \lambda_{0} u_{0}-(2+\alpha) F\left(\lambda_{0} u_{0}\right)\right] \\
& +\frac{\mu_{0}^{2} \alpha}{2} \int_{\Omega}\left|\nabla v_{0}\right|^{2}+\int_{\Omega}\left[f\left(\mu_{0} v_{0}\right) \mu_{0} v_{0}-(2+\alpha) F\left(\mu_{0} v_{0}\right)\right] .
\end{aligned}
$$


Observe that the weak convergence of $\left(u_{\varepsilon}, v_{\varepsilon}\right) \in \mathcal{N}_{\varepsilon}$ to $\left(u_{0}, v_{0}\right)$ implies

$$
\begin{aligned}
& \int_{\Omega}\left|\nabla u_{0}\right|^{2}-\int_{\Omega} f\left(u_{0}\right) u_{0}+\lim _{\varepsilon \rightarrow 0} \varepsilon^{-1} \int_{\Omega} G_{u}\left(u_{\varepsilon}, v_{\varepsilon}\right) u_{\varepsilon} \leqslant 0, \\
& \int_{\Omega}\left|\nabla v_{0}\right|^{2}-\int_{\Omega} f\left(v_{0}\right) v_{0}+\lim _{\varepsilon \rightarrow 0} \varepsilon^{-1} \int_{\Omega} G_{v}\left(u_{\varepsilon}, v_{\varepsilon}\right) v_{\varepsilon} \leqslant 0, \\
& \lim _{\varepsilon \rightarrow 0} c_{\varepsilon} \geqslant \frac{1}{2} \int_{\Omega}\left(\left|\nabla u_{0}\right|^{2}+\left|\nabla v_{0}\right|^{2}\right)-\int_{\Omega} F\left(u_{0}\right)-\int_{\Omega} F\left(v_{0}\right)+\lim _{\varepsilon \rightarrow 0} \varepsilon^{-1} \int_{\Omega} G\left(u_{\varepsilon}, v_{\varepsilon}\right) .
\end{aligned}
$$

As before, multiply the last inequality by $(2+\alpha)$ and then sum up the three

$$
\begin{aligned}
(2+\alpha) \lim _{\varepsilon \rightarrow 0} c_{\varepsilon} \geqslant & \frac{\alpha}{2} \int_{\Omega}\left(\left|\nabla u_{0}\right|^{2}+\left|\nabla u_{0}\right|^{2}\right) \\
& +\int_{\Omega}\left[f\left(u_{0}\right) u_{0}-(2+\alpha) F\left(u_{0}\right)\right]+\int_{\Omega}\left[f\left(v_{0}\right) v_{0}-(2+\alpha) F\left(v_{0}\right)\right] \\
& +\lim _{\varepsilon \rightarrow 0} \varepsilon^{-1} \int_{\Omega}\left[(2+\alpha) G\left(u_{\varepsilon}, v_{\varepsilon}\right)-G_{u}\left(u_{\varepsilon}, v_{\varepsilon}\right) u_{\varepsilon}-G_{v}\left(u_{\varepsilon}, v_{\varepsilon}\right) v_{\varepsilon}\right] .
\end{aligned}
$$

Now, since by construction $c_{0} \geqslant c_{\varepsilon}$ for all $\varepsilon>0$, we can compare this inequality with (17); to shorten notation we introduce $\mathcal{F}(t):=f(t) t-(2+\alpha) F(t)$, noting that $\mathcal{F}(t)$ is increasing for $t>0$ by $\left(f_{2}\right)$. It turns out

$$
\begin{aligned}
& \frac{\alpha}{2} \int_{\Omega}\left[\left(\lambda_{0}^{2}-1\right)\left|\nabla u_{0}\right|^{2}+\left(\mu_{0}^{2}-1\right)\left|\nabla v_{0}\right|^{2}\right] \\
& \quad+\int_{\Omega}\left[\mathcal{F}\left(\lambda_{0} u_{0}\right)-\mathcal{F}\left(u_{0}\right)\right]+\int_{\Omega}\left[\mathcal{F}\left(\mu_{0} v_{0}\right)-\mathcal{F}\left(v_{0}\right)\right] \\
& \quad \geqslant \lim _{\varepsilon \rightarrow 0} \varepsilon^{-1} \int_{\Omega}\left[(2+\alpha) G\left(u_{\varepsilon}, v_{\varepsilon}\right)-G_{u}\left(u_{\varepsilon}, v_{\varepsilon}\right) u_{\varepsilon}-G_{v}\left(u_{\varepsilon}, v_{\varepsilon}\right) v_{\varepsilon}\right] .
\end{aligned}
$$

By assumptions $\left(h_{2}\right),\left(h_{3}\right)$ it turns out that the term at the r.h.s. is non-negative: now since both $\lambda_{0}$ and $\mu_{0}$ are not greater than 1 , we conclude that in fact $\lambda_{0}=\mu_{0}=1$. This proves assertion (i) and, in turn, implies that:

$$
\lim _{\varepsilon \rightarrow 0} \varepsilon^{-1} \int_{\Omega}\left[(2+\alpha) G\left(u_{\varepsilon}, v_{\varepsilon}\right)-G_{u}\left(u_{\varepsilon}, v_{\varepsilon}\right) u_{\varepsilon}-G_{v}\left(u_{\varepsilon}, v_{\varepsilon}\right) v_{\varepsilon}\right]=0 .
$$

From this the strong convergence of $u_{\varepsilon}$ and $v_{\varepsilon}$ to their limits easily follows. Indeed, if we assume for instance that $\lim \int_{\Omega}\left|\nabla u_{\varepsilon}\right|^{2}>\int_{\Omega}\left|\nabla u_{0}\right|^{2}$, then all the above inequalities become strict, leading a contradiction in (19). Thus (ii) is proved.

Let us now prove assertion (iii). To this aim we deduce by (16) and $\lambda_{0}=1$, the relation $\int_{\Omega}\left|\nabla u_{0}\right|^{2}=\int_{\Omega} f\left(u_{0}\right) u_{0}$. Back to (18) we obtain that

$$
\lim _{\varepsilon \rightarrow 0} \varepsilon^{-1} \int_{\Omega} G_{u}\left(u_{\varepsilon}, v_{\varepsilon}\right) u_{\varepsilon}=0
$$


and the analogs for $v_{0}$. Now (19) becomes

$$
\lim _{\varepsilon \rightarrow 0} \varepsilon^{-1} \int_{\Omega} G\left(u_{\varepsilon}, v_{\varepsilon}\right)=0
$$

as desired. Furthermore, we deduce by (iii) that

$$
\lim _{\varepsilon \rightarrow 0} c_{\varepsilon}=J\left(u_{0}, v_{0}\right) \geqslant c_{0},
$$

where the last inequality follows by the definition of $c_{0}$ and (i). Now it is enough to recall that $\lim _{\varepsilon \rightarrow 0} c_{\varepsilon} \leqslant c_{0}$ by (14) to show that indeed $\lim _{\varepsilon \rightarrow 0} c_{\varepsilon}=c_{0}$, proving (iv).

Proof of 2). - In order to conclude the proof of Proposition 4.1 it suffices to show that $u_{0}$ and $v_{0}$ have disjoint support: this is consequence of property (iii) and of the strong convergence of $\left(u_{\varepsilon}, v_{\varepsilon}\right)$ to its weak limit. Indeed this implies

$$
\int_{\Omega} G\left(u_{0}, v_{0}\right)=0
$$

by assumption $\left(h_{3}\right)$ this means $u_{0}(x) \cdot v_{0}(x)=0$ a.e. $x \in \Omega$ and proves $\operatorname{supp}\left(u_{0}\right) \cap$ $\operatorname{supp}\left(v_{0}\right)=\emptyset$. Moreover by (iv) we also obtain $J_{0}\left(u_{0}, v_{0}\right)=c_{0}$, finally proving that $\left(u_{0}, v_{0}\right)$ solves $\left(P_{0}\right)$.

As a final remark, note that, if we pass to the limit in (2) as $\varepsilon \rightarrow 0$, by exploiting the strong convergence of the solutions $\left(u_{\varepsilon}, v_{\varepsilon}\right)$ to the pair $\left(u_{0}, v_{0}\right)$ and (iii), we realize that

$$
\begin{aligned}
& -\Delta u_{0}(x)=f\left(u_{0}(x)\right) \quad x \in \operatorname{supp}\left(u_{0}\right), \\
& -\Delta v_{0}(x)=f\left(v_{0}(x)\right) \quad x \in \operatorname{supp}\left(v_{0}\right) .
\end{aligned}
$$

\section{An equivalent problem}

Let $\left(u_{0}, v_{0}\right)$ be the pair solving $\left(P_{0}\right)$ as provided by Proposition 4.1. Aim of this section is to prove that $w_{0}=u_{0}-v_{0}$ is a solution of (3) as in Theorem 2.3. We have already noticed in the introduction that this result leads to answer our main questions about the original Nehari's problem (1).

The proof of Theorem 2.3 is based on the equivalence between the approximating procedure shown above and a minimax technique of Nehari type devoted to prove the existence of a changing sign solution for the Eq. (3).

\subsection{A changing sign solution of (3)}

Let us consider the energy functional associated to (3):

$$
J_{*}(w):=\int_{\Omega}\left(\frac{1}{2}|\nabla w(x)|^{2}-F(x, w(x))\right) \mathrm{d} x, \quad w \in H_{0}^{1}(\Omega) .
$$


Since we are interested in functions of $H_{0}^{1}(\Omega)$ which change sign, it is worth introducing the subset

$$
\mathcal{T}_{*}:=\left\{u \in H_{0}^{1}(\Omega): u^{+} \not \equiv 0, u^{-} \not \equiv 0\right\} .
$$

Let us define

$$
\mathcal{N}_{*}:=\left\{u \in \mathcal{T}_{*}: \nabla J\left(u^{+}\right) \cdot u^{+}=\nabla J\left(u^{-}\right) \cdot u^{-}=0\right\}
$$

and the minimum of $J_{*}$ restricted to $\mathcal{N}_{*}$

$$
c_{*}:=\inf _{u \in \mathcal{N}_{*}} J_{*}(u) .
$$

We claim that:

PROPOSITION 5.1. - There exists a changing sign function $w_{*} \in H_{0}^{1}(\Omega)$ such that $J_{*}\left(w_{*}\right)=c_{*}$ and

$$
-\Delta w_{*}(x)=f\left(x, w_{*}(x)\right) \quad x \in \Omega .
$$

The proof of this existence theorem follows the line and the technical ideas exploited in Section 3 and thus it falls naturally in three parts corresponding to Lemmas 3.1, 3.2 and 3.3:

LEMMA 5.1. - Let $u \in \mathcal{T}_{*}$ and consider

$$
\Phi(u):=\sup _{\substack{s>0 \\ t>0}} J_{*}\left(s u^{+}-t u^{-}\right) .
$$

Then there exists a unique pair of positive numbers $\lambda^{+}(u)$ and $\lambda^{-}(u)$ such that

(i) $\Phi(u)=J_{*}\left(\lambda^{+}(u) u^{+}-\lambda^{-}(u) u^{-}\right)$;

(ii) $\lambda^{+}(u) u^{+}-\lambda^{-}(u) u^{-} \in \mathcal{N}_{*}$;

(iii) there exist $r_{1}, r_{2}>0$ such that if $u \in \mathcal{N}_{*}$ then $\left\|u^{ \pm}\right\|_{p} \geqslant r_{1},\left\|u^{ \pm}\right\| \geqslant r_{2}$;

(iv) $\Phi(\cdot)$ is lower semicontinuous with respect to the weak convergence in $\mathcal{T}_{*}$;

(v) the map $u \mapsto\left(\lambda^{+}(u), \lambda^{-}(u)\right)$ is continuous from $H_{0}^{1}(\Omega)$ to $\mathbb{R} \times \mathbb{R}$.

As an immediate consequence of this lemma, it turns out that

$$
d_{*}:=\inf _{u \in \mathcal{T}_{*}} \Phi(u) \equiv c_{*} ;
$$

this fact is crucial when proving that the problem of minimizing $J_{*}$ on the Nehari manifold $\mathcal{N}_{*}$ has a solution:

LEMMA 5.2. - There exists $u_{*} \in \mathcal{N}_{*}$ such that $J\left(u_{*}\right)=c_{*}$.

Finally, the third step in the proof of the existence of a changing sign solution to (3) consists in proving that any point at level $c_{*}$ is critical for $J_{*}$ :

LEMMA 5.3. - If $u_{*} \in \mathcal{N}_{*}$ and $J_{*}\left(u_{*}\right)=c_{*}$, then $u_{*}$ is a critical point of $J_{*}$.

The following remark allows us to get easily the proofs of the first two lemmas directly from the corresponding results in Section 3. As a matter of facts it is equivalent to study 
$J_{*}$ on $H_{0}^{1}(\Omega)$ and $J_{0}$ on $H_{0}^{1}(\Omega) \times H_{0}^{1}(\Omega)$ restricted to pairs $(u, v)$ such that

$$
\operatorname{supp}(u) \cap \operatorname{supp}(v)=\emptyset
$$

since it holds

$$
\forall w \in H_{0}^{1}(\Omega) \quad u:=w^{+} v:=w^{-} \Rightarrow\left\{\begin{array}{l}
J_{*}(w)=J_{0}(u, v) \\
\nabla J_{*}(w)=\nabla J_{0}(u, v) .
\end{array}\right.
$$

Note that the constraint (22) is closed both with respect to strong and weak convergence of $H_{0}^{1}(\Omega) \times H_{0}^{1}(\Omega)$; with this we conclude that Lemmas 5.1 and 5.2 holds as simple consequence of Lemmas 3.1 and 3.2.

Concerning the proof of Lemma 5.3, we point out that it formally follows the scheme illustrated in Lemma 3.3, but, for the readers convenience, we prefer to discuss it in all the details.

Proof of Lemma 5.3. - Assume by contradiction that $u_{*} \in \mathcal{N}_{*}, \quad J_{*}\left(u_{*}\right)=c_{*}$, but $\nabla J_{*}\left(u_{*}\right) \neq 0$. Then there exists $\rho>0$ and $\delta>0$ (we may assume $4 \delta<r_{2}$ as in Lemma 5.1(iii)) such that

$$
u \in B_{\delta}\left(u_{*}\right) \Rightarrow\|\nabla J(u)\| \geqslant \rho .
$$

By the quantitative deformation lemma (see, for instance, [24]) we derive the existence of a continuous map $\eta: H_{0}^{1}(\Omega) \rightarrow H_{0}^{1}(\Omega)$ and a constant $\varepsilon>0$ such that:

(i) $\eta(u)=u$ for all $u$ such that $\left\|u-u_{*}\right\|>4 \delta$ or $\left|J_{*}(u)-c_{*}\right|>2 \varepsilon$;

(ii) $J_{*}(\eta(u)) \leqslant J_{*}(u)$ for all $u \in H_{0}^{1}(\Omega)$;

(iii) $u \in B_{2 \delta}\left(u_{*}\right) \wedge J_{*}(u) \leqslant c_{*}+\varepsilon \Rightarrow J_{*}(\eta(u)) \leqslant c_{*}-\varepsilon$.

Let us consider the deformation under this map of the surface $\Gamma(s, t):=s R u_{*}^{+}-t R u_{*}^{-}$, where $R$ is fixed in such a way that $(R-1) r_{2}>4 \delta$. By the monotonicity properties of $\eta$ and Lemma 5.1, we have that $\sup _{s>0, t>0} \eta(\Gamma(s, t))<c_{*}$. Now we claim that $\eta \circ \Gamma$ intersects the manifold $\mathcal{N}_{*}$ and thus, by definition of $c_{*}$ it holds $\sup \eta(\Gamma(s, t)) \geqslant c_{*}$, a contradiction. To prove the claim, let us consider the map:

$$
\begin{aligned}
\mathcal{H}:[0,1]^{2} & \rightarrow \mathbb{R} \times \mathbb{R} \\
(s, t) & \mapsto\left(\lambda^{+}(\eta(\Gamma(s, t)))-1, \lambda^{-}(\eta(\Gamma(s, t)))-1\right)
\end{aligned}
$$

which is continuous by Lemma 5.1(v). By construction it holds:

- if $s=0$, then $\mathcal{H}_{1}(0, \cdot)=+\infty$; indeed, since $\Gamma(0, t)=-t u_{*}^{-}$, it holds $\| \Gamma(0, t)^{+}-$ $u_{*}^{+}\|=\| u_{*}^{+} \|>r_{2}>4 \delta$ by the choice of $\delta$. Thus by property (i) of the map $\eta$, we know that $\eta(\Gamma(0, t))=\Gamma(0, t)=$ and thus $\mathcal{H}_{1}(0, t)=\lambda^{+}\left(-t u_{*}^{-}\right)=+\infty$;

- if $s=1$, then $\mathcal{H}_{1}(0, \cdot)<0$; indeed since $\Gamma(1, t)=R u_{*}^{+}-t R u_{*}^{-}$, it holds $\left\|\Gamma(1, t)^{+}-u_{*}^{+}\right\|=(R-1)\left\|u_{*}^{+}\right\|>4 \delta$ by the choice of $R$. Thus $\eta(\Gamma(1, t))=\Gamma(1, t)$ and $\mathcal{H}_{1}(1, t)=\lambda^{+}\left(R u_{*}^{+}-t R u_{*}^{-}\right)<1$.

Analogous considerations allow to prove that the second component of $\mathcal{H}$ is positive when $t=0$ and negative if $t=1$. Thus we are in condition to apply Miranda Theorem [21], and we find $(\bar{s}, \bar{t})$ such that $\mathcal{H}(\bar{s}, \bar{t})=(0,0)$; this implies $\eta(\Gamma(\bar{s}, \bar{t})) \in \mathcal{N}_{*}$, that concludes the proof. 
We have thus proved the existence result in Proposition 5.1. The remainder of this section will be devoted to establish the equivalence between the variational procedure on which the proof of Proposition 5.1 is based and the approximating technique leading to define $c_{0}$ as in Section 4. This is stated in the following:

Lemma 5.4. - Let $c_{0}$ and $c_{*}$ be defined as in (12) and (21). Then $c_{0} \equiv c^{*}$ and setting $w_{0}:=u_{0}-v_{0}$ with $\left(u_{0}, v_{0}\right)$ as in (15), then $w_{0}$ is a solution of (3). Moreover $\left(\operatorname{supp}\left(u_{0}\right) \cup \operatorname{supp}\left(v_{0}\right)\right)$ is a solution of $(1)$.

Proof. - let us remark that, if $u$ and $v$ have disjoint supports, then $J_{0}(u, v)=J_{*}(u)+$ $J_{*}(v)=J_{*}(u-v)$.

Let $w_{*}$ as in Theorem 5.1 and define $u_{*}:=w_{*}^{+}$and $v_{*}:=w_{*}^{-}:$with these we will prove the first inequality $c_{0} \leqslant c^{*}$ : since $w_{*}$ is critical for $J_{*}$ one easily finds

$$
\frac{\partial J_{0}\left(u_{*}, v_{*}\right)}{\partial u} \cdot u_{*}=\frac{\partial J_{0}\left(u_{*}, v_{*}\right)}{\partial v} \cdot v_{*}=0
$$

and hence $\left(u_{*}, v_{*}\right) \in \mathcal{N}_{0}$. Since $c_{0}=\inf _{\mathcal{N}_{0}} J_{0}(u, v)$, then $c_{0} \leqslant J_{0}\left(u_{*}, v_{*}\right)=J_{*}\left(w_{*}\right)=c_{*}$, proving the desired inequality.

$c_{0} \geqslant c_{*}$ : now let us consider the pair $\left(u_{0}, v_{0}\right)$ as in Proposition 4.1. Since $\left(u_{0}, v_{0}\right) \in \mathcal{N}_{0}$, then $w_{0}:=u_{0}-v_{0}$ belongs to $\mathcal{N}_{*}$ and $J_{*}\left(w_{0}\right)=J_{0}\left(u_{0}, v_{0}\right)=c_{0}$. This implies $c_{*} \leqslant c_{0}$ by definition of $c_{*}$.

Now we know that $J_{*}\left(w_{0}\right)=c_{*}$, and it suffices to apply Lemma 5.2 to prove that $w_{0}$ is indeed a critical point for $J_{*}$. Moreover by the regularity theory for elliptic PDEs we know that $\operatorname{supp}\left(u_{0}\right)$ and $\operatorname{supp}\left(v_{0}\right)$ are open and thus they provide a solution to (1).

With this we have finally proven the crucial Theorem 2.3 .

\section{REFERENCES}

[1] T. Bartsch, Z.Q. Wang, On the existence of changing sign solutions for semilinear Dirichlet problem, Topol. Methods Nonlinear Anal. 7 (1997) 115-131.

[2] T. Bartsch, Z.Q. Wang, Existence and multiplicity results for some superlinear elliptic problem on $\mathbb{R}^{n}$, Comm. Partial Differential Equations 20 (1995) 1725-1741.

[3] T. Bartsch, M. Willem, Infinitely many radial solutions of a semilinear elliptic problem on $\mathbb{R}^{N}$, Arch. Rat. Mech. Anal. 124 (1993) 261-276.

[4] A. Castro, J. Cossio, J.M. Neuberger, A sign-changing solution for a superlinear Dirichlet problem, Rocky Mountain J. Math. 27 (1997) 1041-1053.

[5] A. Castro, J. Cossio, J.M. Neuberger, A minimax principle, index of critical point, and existence of sign changing solutions to elliptic boundary value problems, Electron. J. Differential Equations 2 (1998).

[6] C. Cosner, A. Lazner, Stable coexistence in the Volterra-Lotka competition model with diffusion, SIAM J. Math. Anal. 44 (1984) 1112-1132.

[7] M. Conti, L. Merizzi, S. Terracini, Remarks on variational methods and lower-upper solutions, NoDEA 6 (1999) 371-393.

[8] E.N. Dancer, Competing species systems with diffusion and large interaction, Rend. Sem. Mat. Fis. Milano 65 (1995) 23-33.

[9] E.N. Dancer, On positive solutions of some pairs of differential equations, Trans. Amer. Math. Soc. 284 (1984) 729-743. 
[10] E.N. Dancer, On positive solutions of some pairs of differential equations, II, J. Differential Equations 60 (1985) 236-258.

[11] E.N. Dancer, On the existence and uniqueness of positive solutions for competing species models with diffusion, Trans. Amer. Math. Soc. 326 (1991) 829-859.

[12] E.N. Dancer, A counterexample on competing species equations, Differential Integral Equations 9 (1996) 239-246.

[13] E.N. Dancer, On uniqueness and stability for solutions of singularly perturbed predatorprey type equations with diffusion, J. Differential Equations 102 (1993) 1-32.

[14] E.N. Dancer, Y.H. Du, On sign-changing solutions of certain semilinear elliptic problems, Appl. Anal. 56 (1995) 193-206.

[15] E.N. Dancer, Y.H. Du, Positive solutions for a three-species competition system with diffusion. I. General existence results, Nonlinear Anal. 24 (1995) 337-357.

[16] E.N. Dancer, Y.H. Du, Positive solutions for a three-species competition system with diffusion. II. The case of equal birth rates, Nonlinear Anal. 24 (1995) 359-373.

[17] E.N. Dancer, Y.H. Du, Competing species equations with diffusion, large interactions, and jumping nonlinearities, J. Differential Equations 114 (1994) 434-475.

[18] E.N. Dancer, Z.M. Guo, Uniqueness and stability for solutions of competing species equations with large interactions, Comm. Appl. Nonlinear Anal. 1 (1994) 19-45.

[19] P. Korman, A. Leung, On the existence and uniqueness of positive steady states in LotkaVolterra ecological models with diffusion, Appl. Anal. 26 (1987) 145-160.

[20] A.C. Lazer, P.J. McKenna, On steady state solutions of a system of reaction-diffusion equations from biology, Nonlinear Anal. TMA 6 (1982) 523-530.

[21] C. Miranda, Un'osservazione sul teorema di Brouwer, Boll. U.M.I. Serie II, Anno II 1 (1940) 5-7.

[22] Z. Nehari, Characteristic values associated with a class of nonlinear second order differential equations, Acta Math. 105 (1961) 141-175.

[23] Z.Q. Wang, On a superlinear elliptic equation, Ann. Inst. H. Poincaré Anal. Non Linéaire 8 (1) (1991) 43-57.

[24] M. Willem, Minimax Theorems, Birkhäuser, 1996. 